\title{
Low-Complexity ICI Suppression Methods Utilizing Cyclic Prefix for OFDM Systems in High-Mobility Fading Channels
}

\author{
Chun-Ying Ma, Student Member, IEEE, Sheng-Wen Liu, and Chia-Chi Huang
}

\begin{abstract}
This paper focuses on a simple intercarrier interference (ICI) suppression scheme that linearly combines the intersymbol interference (ISI)-free part of a cyclic prefix (CP) and its corresponding part in an orthogonal frequency-division multiplexing (OFDM) signal to soothe the ICI effect caused by a timevarying channel. In contrast with existing heuristic combining weights in the literature for ICI suppression, we derived three sets of optimum combining weights in different aspects. Through simulations, we show that the proposed combining weights outperform other existing heuristic combining weights. In addition, a simplified implementation is introduced to reduce the computational complexity of combining. Furthermore, the proposed methods can be incorporated with other ICI mitigation methods to further improve the system performance.
\end{abstract}

Index Terms-Cyclic prefix (CP) combining, cyclic prefix (CP) recycling, high mobility, intercarrier interference (ICI) suppression, low-complexity ICI mitigation method, orthogonal frequency-division multiplexing (OFDM), sliding window.

\section{INTRODUCTION}

$\mathbf{O}$ RTHOGONAL frequency-division multiplexing (OFDM) is a promising modulation scheme mainly due to its robustness against frequency-selective channels. The main idea is to divide a broadband frequency-selective channel into a number of narrowband flat subchannels using fast Fourier transform (FFT). By inserting a cyclic prefix (CP) before transmission, intersymbol interference (ISI) is avoided, and the orthogonality among subcarriers is preserved. As a result, the distortion caused by the channel effect can be equalized merely by a one-tap equalizer on each subcarrier. Owing to its immunity against frequency-selective channels, OFDM has become a key air interface for modern wireless communication systems such as digital video broadcasting (DVB), Long Term Evolution Advanced (LTE-A), and wireless local area networks.

Manuscript received March 6, 2013; revised July 9, 2013; accepted August 4, 2013. Date of publication August 15, 2013; date of current version February 12, 2014. This work was supported by the National Science Council of the Republic of China under Grant NSC 100-2220-E-009-025 and Grant 99-2221-E-009075. The review of this paper was coordinated by Dr. T. Jiang.

C.-Y. Ma and C.-C. Huang are with the Department of Electrical and Computer Engineering, National Chiao Tung University, Hsinchu 300, Taiwan (e-mail: ma_chun_ying.cm93@g2.nctu.edu.tw; huangcc@cc.nctu.edu.tw).

S.-W. Liu was with the Department of Electrical and Computer Engineering, National Chiao Tung University, Hsinchu 300, Taiwan. He is now with Novatek Microelectronics Corporation, Hsinchu 300, Taiwan (e-mail: skykid06@gmail.com).

Digital Object Identifier 10.1109/TVT.2013.2278173
In contrast with its ability to combat channel selectivity in frequency, OFDM is, however, sensitive to channel selectivity in time. The time selectivity of the channel becomes nontrivial when the system operates in a high-mobility environment. In this situation, the orthogonality among subcarriers will be destroyed, and the loss of orthogonality will cause intercarrier interference (ICI), which may severely degrade bit error rate (BER) performance [1].

To combat the ICI effect caused by the time-varying channel, numerous ICI mitigation schemes were proposed [2]-[17]. In [2]-[4], linear equalizers based on minimum-mean-squareerror and least squares (LS) criteria were proposed. In [5][9], methods based on successive interference cancelation were proposed to further improve the performance. A maximum a priori/maximum-likelihood-based joint channel slope estimation and data detection method using an expectationmaximization algorithm was proposed in [10]. Decisionfeedback equalizers were proposed in [6], [11], and [12]. In [13], a method that uses a $Q$-tap equalizer instead of a traditional one-tap equalizer to suppress the ICI power was proposed. In [14], channel estimation and ICI mitigation methods that utilize CP or adjacent OFDM symbols were proposed. In [15]-[17], time-domain windowing techniques for concentrating ICI into its main band were proposed. This technique is also called an ICI shortening technique, and it can cooperate with other equalization techniques such as in [2]-[13]. In [18] and [19], low-complexity joint equalization and channel coding algorithms were proposed.

In this paper, we focus on a simple scheme investigated in [20]-[25], where the ISI-free part of the CP is linearly combined with its corresponding part in the OFDM signal to suppress the ICI power. This method is motivated by the fact that, in some situations, the length of a $\mathrm{CP}$ is much larger than the delay spread; therefore, a considerable number of ISI-free samples exist. For instance, in broadcasting systems, because the length of a CP is chosen to support the worst-case delay spread within its coverage area, the CP length may be much larger than some users' delay spreads. Hence, exploiting these unused resources is an important issue. Furthermore, it is worth noting that this scheme can be incorporated with most common ICI mitigation methods, e.g., in [2]-[13]; that is, the output of $\mathrm{CP}$ combining can be regarded as a new OFDM symbol, whose ICI power is less than the original power, and the methods [2]-[13] can be applied to this new OFDM symbol to further mitigate the ICI effect. 
For the a priori parts in this arena, Chang proposed a pioneer work by extending the length of a CP [20]. He proposed a novel ICI self-cancelation scheme and proved that if the length of a $\mathrm{CP}$ is extended long enough so that the length of ISIfree samples in the $\mathrm{CP}$ is equal to the length of the OFDM symbol, the ICI effect can be completely removed with linear time-varying channel assumption. (This assumption is generally valid at reasonable vehicular speed [14].) Since it has to modify the transmit signal formats, this method is not adaptable to almost all the modern wireless communication systems. In practice, there are only limited ISI-free samples in a CP, whose length is much shorter than the OFDM symbol length. In this situation, the work in [20] suggested to set the combining weights uniformly, but this is apparently a suboptimum solution in the sense of ICI minimization. Afterward, in [21]-[23], heuristic algorithms were proposed to choose better combining weights to improve the performance of [20]. In a parallel work [24], an engineering intuition that Frank function window may be the optimum combining window was shown.

In this paper, contrary to all the heuristic approaches used in the literature [20]-[24], we mathematically analyze the effect of weighted segment combining and propose three sets of optimum combining weights in different aspects. Method 1, which will be shown to be equivalent to [24], is designed to minimize the ICI power. Unlike the engineering approach used in [24], the optimum combining weights are analytically derived in this paper. To take the noise effect into consideration, we propose Method 2, which minimizes the ICI-plus-noise power. Finally, to take advantage of the ISI-polluted CP samples, which are simply discarded in previous works, we propose Method 3.

The following notations are adhered to throughout this paper. Boldface lowercase and uppercase letters denote vectors and matrices, respectively. Superscripts $(\cdot)^{T}$ and $(\cdot)^{H}$ denote the transpose and Hermitian of a matrix or a vector, respectively. Superscript $(\cdot)^{*}$ is the conjugate of a complex variable. The OFDM block length is defined as $N . \mathbf{F}$ is the $N \times N$ unitary discrete Fourier transform (DFT) matrix whose $(m, n)$ th entry is given by $(1 / \sqrt{N}) \exp (-j(2 \pi m n / N))$ $\forall m, n \in\{0,1, \ldots, N-1\}$. We define $\operatorname{diag}\{\mathbf{x}\}$ as a diagonal matrix with vector $\mathrm{x}$ on its diagonal. Modulo- $N$ operation is represented by $(\cdot)_{N}$. Numbers $\mathbf{1}$ and $\mathbf{0}$ represent column vectors with appropriate dimensions containing all ones and all zeros, respectively. We use $\mathbf{O}_{K \times L}$ to denote a $K \times L$ matrix containing all zeros and $\mathbf{I}_{K}$ to denote a $K \times K$ identity matrix, and the subscript may be ignored if there is no ambiguity in the matrix dimension. Statistical expectation is denoted $\mathbb{E}[(\cdot)]$. $\operatorname{tr}(\cdot)$ denotes trace operation. Finally, we use $[\mathbf{x}]_{\mathcal{A}}$ and $[\mathbf{X}]_{(\mathcal{U}, \mathcal{V})}$ to represent a subvector of $\mathbf{x}$ and a submatrix of $\mathbf{X}$ with the corresponding index sets $\mathcal{A}$ and $(\mathbf{U} \times \mathcal{V})$, respectively.

The remainder of this paper is organized as follows. In Section II, we introduce the system model and the highmobility fading channel. Next, in Section III, we propose three methods, solving three different ICI suppression optimization problems. Then, we compare the complexity of the proposed methods in detail in Section IV. Afterward, simulation results are given in Section V. Finally, conclusions are drawn in Section VI.

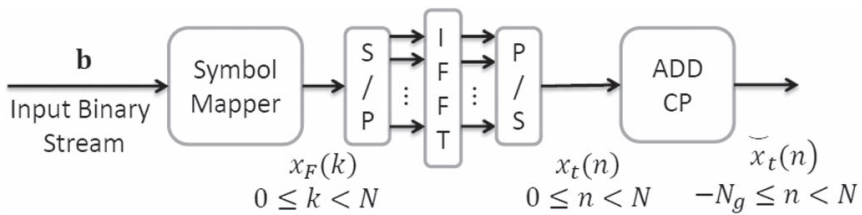

Fig. 1. Block diagram of the OFDM transmitter.

\section{SYSTEM MODEL}

The block diagram of the OFDM transmitter is shown in Fig. 1. Input binary stream $\mathbf{b}$ is fed into a symbol mapper. Symbol mapper output $x_{F}(k)$ is the frequency-domain signal on subcarrier $k$. Without loss of generality, we assume that $\mathbb{E}\left[\left|x_{F}(k)\right|^{2}\right]=1, k=0, \ldots, N-1$. The $N$-point inverse FFT (IFFT) output can be expressed as

$$
x_{t}(n)=\frac{1}{\sqrt{N}} \sum_{k=0}^{N-1} x_{F}(k) e^{\frac{2 \pi k n}{N}}, \quad n=0,1, \ldots, N-1 .
$$

To combat ISI, a CP is inserted before transmission. After the $\mathrm{CP}$ insertion, the equivalent baseband time-domain transmitted signal $\breve{x}_{t}(n)$ can be expressed as

$$
\breve{x}_{t}(n)=x_{t}\left((n)_{N}\right), \quad-N_{g} \leqslant n \leqslant N-1
$$

where $N_{g}$ is the length of the CP. We further assume that the length of the $\mathrm{CP}$ is relatively longer than the maximum delay spread, and there are $q$ samples in the $\mathrm{CP}$ that are not affected by the ISI, i.e., $q \triangleq N_{g}-L+1$. The interval $[-q,-1]$ is commonly known as the ISI-free region [20]-[22], and interval $\left[-N_{g},-q-1\right]$ is named the ISI-polluted region. Received signal $y_{t}(n)$ can be expressed as

$y_{t}(n)=\sum_{l=0}^{L-1} h^{(l)}(n) x_{t}\left((n-l)_{N}\right)+\varepsilon(n), \quad n=-q, \ldots, N-1$

where $L-1$ is the maximum path delay, $h^{(l)}(n)$ is the complex channel gain at time lag $l$ and sample time $n$, and $\varepsilon(n)$ is the time-domain complex additive white Gaussian noise with variance $\sigma^{2}$. The actual path delays can be collected to form set $\mathcal{L}$. Typically, the number of elements in $\mathcal{L}$ is much smaller than $L$, and $h^{(l)}(n)$ equals zeros if $l \notin \mathcal{L}$.

For simplicity in analysis, we define the term segment as follows.

Definition 1: Time-domain received segment $d$ or $\mathbf{y}_{t}^{(d)}$ is defined as

$$
\mathbf{y}_{t}^{(d)} \triangleq\left[\begin{array}{llll}
y_{t}(-d) & y_{t}(1-d) & \cdots & y_{t}(N-1-d)
\end{array}\right]^{T} .
$$

Note that we have $q+1$ ISI-free OFDM segments, namely, $\mathbf{y}_{t}^{(0)}, \ldots, \mathbf{y}_{t}^{(q)}$. The core idea of this paper is to linearly combine these segments to form a new OFDM symbol to mitigate the ICI effect induced by the time-varying channel.

The corresponding frequency-domain received segment $\mathbf{y}_{F}^{(d)}$ is defined as

$$
\mathbf{y}_{F}^{(d)} \triangleq \mathbf{F} \mathbf{y}_{t}^{(d)}
$$




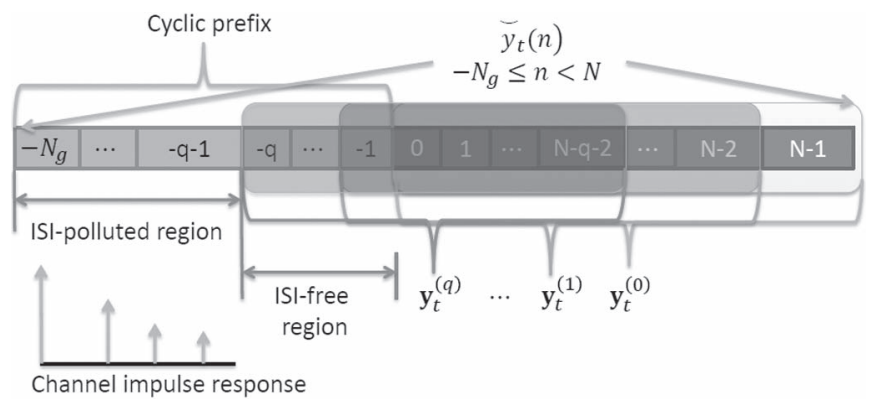

Fig. 2. The ISI-free region and OFDM signal segments.

In brief, the relationship between time-domain received segments is shown in Fig. 2.

We use the traditional Jakes' model [35] to model the timevarying effect, and it is reported in [4], [10], [14], and [24] that a piecewise-linear approximation to the channel variation is sufficient at reasonable vehicular speed. Therefore, in the following analysis, we approximate each time-varying channel path $h^{(l)}(p)$ within one OFDM symbol period by a linear function, i.e.,

$$
h^{(l)}(p) \approx h^{(l)}(0)+p \cdot a^{(l)} \quad \forall p \in\left[-N_{g}, N-1\right] \quad \forall l \in \mathcal{L}
$$

where $a^{(l)} \in \mathbb{C}$ is the channel variation slope of the path at lag $l$. We define $h_{\text {mid }}^{(l)}$ as the complex channel gain of the path at time lag $l$ in the middle of the original OFDM signal, i.e.,

$$
h_{\text {mid }}^{(l)} \triangleq \frac{1}{2}\left(h^{(l)}\left(\frac{N}{2}-1\right)+h^{(l)}\left(\frac{N}{2}\right)\right)
$$

and the corresponding frequency-domain channel $H_{\text {mid }}(k)$ is defined as

$$
H_{\text {mid }}(k) \triangleq \sum_{l \in \mathcal{L}} h_{\text {mid }}^{(l)} e^{-\jmath \frac{2 \pi k l}{N}}, \quad k=0,1, \ldots, N-1 .
$$

We also define the frequency-domain channel slope $w(k)$ as

$$
w(k) \triangleq \sum_{l \in \mathcal{L}} a^{(l)} e^{-\jmath \frac{2 \pi k l}{N}}, \quad k=0,1, \ldots, N-1 .
$$

Afterward, we can represent the original (segment 0) frequency-domain signal $y_{F}^{(0)}(k)$ as [10], [14]

$y_{F}^{(0)}(k)=\sum_{m=0}^{N-1} x_{F}(m) H^{(0)}(k, m)+e^{(0)}(k) \quad \forall k \in[0, N-1]$

where $e^{(0)}(k)$ is the frequency-domain additive noise of the received segment 0 on subcarrier $k$

$$
\begin{aligned}
H^{(0)}(k, m) & = \begin{cases}H_{\operatorname{mid}}(k), & \text { if } k=m \\
w(m) \Phi(k-m), & \text { if } k \neq m\end{cases} \\
\Phi(r) & =-1 /\left(1-\exp \left(-\jmath \frac{2 \pi r}{N}\right)\right), \quad r \neq 0 .
\end{aligned}
$$

The time-domain received signal of segment $d$, i.e., $\mathbf{y}_{t}^{(d)}$, can be regarded as the received samples contributed from the timedomain OFDM samples $\left\{\breve{x}_{t}(n)\right\}_{n=-q}^{N-q-1}$, which is the $q$-step rightward circularly shifted version of the original transmitted samples. Consequently, the equivalent frequency-domain transmit signal of segment $d$ on subcarrier $k$, which is denoted $X_{F}^{(d)}(k)$, can be represented as $X_{F}^{(d)}(k)=X_{F}(k) \times$ $\exp \{-\jmath 2 \pi d k / N\}$. In addition, each channel path of segment $d$ has the same variation slope in time as that of segment 0 , but the complex channel gain of the path at time lag $l$ in the middle of segment $d$ becomes $h_{\text {mid }}^{(l)}-d \cdot a^{(l)}$ rather than $h_{\text {mid }}^{(l)}$. As a result, we can represent the frequency-domain signal of segment $d$, i.e., $y_{F}^{(d)}(k)$, as

$$
\begin{array}{r}
y_{F}^{(d)}(k)=\sum_{m=0}^{N-1} x_{F}(m) H^{(d)}(k, m) e^{-\jmath \frac{2 \pi m d}{N}}+e^{(d)}(k), \\
\forall k \in[0, N-1]
\end{array}
$$

where $e^{(d)}(k)$ is the frequency-domain additive noise of received segment $d$ in subcarrier $k$, and

$$
H^{(d)}(k, m)= \begin{cases}H_{\mathrm{mid}}(k)-d \cdot w(k), & \text { if } k=m \\ w(m) \Phi(k-m), & \text { if } k \neq m .\end{cases}
$$

\section{Proposed Methods}

\section{A. Review of the Existing Methods}

In [20]-[22], the $q+1$ ISI-free segments are weighted and combined to mitigate the ICI effect. The frequency-domain combined signal on subcarrier $k$, which is denoted $v(k)$, can be represented as

$$
v(k)=\sum_{d=0}^{q} u_{d} y_{F}^{(d)}(k) e^{\frac{2 \pi k d}{N}}, \quad k=0,1, \ldots, N-1
$$

where $u_{d}$ is the combining weight of segment $d$, and constraint $\sum_{d=0}^{q} u_{d}=1$ is set for normalization.

In [20], the weights are set uniformly, i.e., $u_{d}=1 /(q+1)$, $\forall d \in[0, q]$, and it is proven that, when $q=N-1$, the ICI effect can be completely canceled. In [21] and [22], heuristic combining weights selection methods were proposed, aiming to cancel the ICI effect induced by the adjacent subcarriers. The combining weights of [21] are given as

$$
u_{d}= \begin{cases}1 / 2, & \text { if } d=0, q \\ 0, & \text { otherwise }\end{cases}
$$

It is also shown through simulations that the method in [21] outperforms the method in [20] if the length of the ISI-free region is less than $N / 2$. Nevertheless, the optimum combining weights have not been studied until the time that we published our previous paper [25], to the best of our knowledge. Recently, in a parallel work [24], the authors have shown from a purely engineering intuition that the optimum weights follow the Frank function window. In the following, we propose three methods to obtain the combining weights, which are optimum in three different aspects. Furthermore, we will show that, the optimum combining weights of our proposed Method 1, although derived analytically, are identical to the Frank function window [24]. 


\section{B. Method 1: ICI Power Minimization}

Here, we derive the optimum combining weights that minimize the ICI power after segment combining. The combined signal $v(k)$ in (15) can be formulated as

$$
\begin{aligned}
& v(k)=x_{F}(k) \sum_{d=0}^{q} u_{d} H^{(d)}(k, k) \\
& +\sum_{\substack{m=0 \\
m \neq k}}^{N-1} x_{F}(m) w(m) \Phi(k-m) \mathbf{t}_{m-k}^{H} \mathbf{u}+z(k)
\end{aligned}
$$

where $\mathbf{u} \triangleq\left[\begin{array}{llll}u_{0} & u_{1} & \cdots & u_{q}\end{array}\right]^{T}$, and

$$
\mathbf{t}_{m-k} \triangleq\left[\begin{array}{llll}
1 & e^{-\jmath \frac{2 \pi(m-k) \cdot 1}{N}} & \cdots & e^{-\jmath \frac{2 \pi(m-k) \cdot q}{N}}
\end{array}\right]^{H}
$$

is the vector that compensates the linear phase shift. We can rewrite (17) as

$$
v(k)=x_{F}(k) \tilde{H}_{\text {mid }}(k)+b(k)+z(k)
$$

where

$$
\tilde{H}_{\text {mid }}(k) \triangleq H_{\text {mid }}(k)+w(k)\left[\begin{array}{llll}
0 & -1 & \cdots & -q
\end{array}\right] \mathbf{u}
$$

is the equivalent channel frequency response of the combined signal

$$
b(k)=\sum_{\substack{m=0 \\ m \neq k}}^{N-1} x_{F}(m) w(m) \Phi(k-m) \mathbf{t}_{m-k}^{H} \mathbf{u}
$$

is the equivalent ICI term after segment combining, and $z(k)$ is the equivalent noise term of the combined signal.

The expected variance of the ICI term is

$\mathbb{E}\left[|b(k)|^{2}\right]=\mathbf{u}^{H} \mathbb{E}\left[\sum_{\substack{m=0 \\ m \neq k}}^{N-1}\left|x_{F}(m)\right|^{2}|w(m)|^{2}|\Phi(k-m)|^{2} \mathbf{T}_{m-k}\right] \mathbf{u}$

where $\mathbf{T}_{m-k} \triangleq \mathbf{t}_{m-k} \mathbf{t}_{m-k}^{H}$. To obtain $\mathbb{E}\left[|w(m)|^{2}\right]$, we treated the time-varying channel as a first-order autoregressive model. In $[10], \mathbb{E}\left[\mathbf{w w}^{H}\right]$ is derived as

$$
\mathbf{C}_{\mathbf{w} \mathbf{w}}=\mathbb{E}\left[\mathbf{w w}^{H}\right]=\mathbf{F C}_{\mathbf{a a}} \mathbf{F}^{H}
$$

where $\mathbf{C}_{\mathbf{a a}}$ equals to $\left(2(1-\beta) /(N-1)^{2}\right) \boldsymbol{\Xi}, \boldsymbol{\Xi}$ is the timedomain channel covariance matrix whose $l$ th diagonal entry $\Xi_{l}$ is the intensity of the power delay profile (PDP) at lag $l, \beta=$ $J_{0}\left(2 \pi f_{D}(N-1) / N\right), J_{0}(\cdot)$ is the zeroth-order Bessel function of the first kind, and $f_{D}$ is the normalized Doppler frequency. The desired term $\mathbb{E}\left[|w(m)|^{2}\right]$ is the $(m, m)$ th entry of $\mathbf{C}_{\mathbf{w w}}$ and is equal to $\left(2(1-\beta) /(N-1)^{2}\right)\left(\sum_{l \in \mathcal{L}} \Xi_{l}\right)$. Without loss of generality, $\sum_{l \in \mathcal{L}} \Xi_{l}$ is normalized to 1 in the following. Therefore, (22) can be expressed as

$$
\mathbb{E}\left[|b(k)|^{2}\right]=\mathbf{u}^{H} \boldsymbol{\Omega} \mathbf{u}
$$

where $\boldsymbol{\Omega} \triangleq\left(2(1-\beta) /(N-1)^{2}\right) \sum_{m=1}^{N-1}|\Phi(m)|^{2} \mathbf{T}_{m}$.
To obtain the optimum combining weights that minimize the ICI power, we have to solve the following optimization problem:

$$
\begin{aligned}
& \min _{\mathbf{u} \in \mathbb{C}^{q+1}} \mathbf{u}^{H} \mathbf{\Omega u} \\
& \text { s.t. } \mathbf{1}^{H} \mathbf{u}=1 .
\end{aligned}
$$

Since the optimization problem (25) is a convex optimization problem, any solution that satisfies the Karush-Kuhn-Tucker (KKT) condition is a global optimum solution [28]. The KKT condition of (25) is given as

$$
\begin{aligned}
\boldsymbol{\Omega} \mathbf{u} & =\lambda \mathbf{1} \\
\mathbf{1}^{T} \mathbf{u} & =1
\end{aligned}
$$

where $\lambda$ is a Lagrange multiplier. If $\Omega$ is invertible, the global optimum solution $\mathbf{u}^{\star}$ of optimization problem (25) is given by

$$
\mathbf{u}^{\star}=\frac{\boldsymbol{\Omega}^{-1} \mathbf{1}}{\mathbf{1}^{H} \boldsymbol{\Omega}^{-1} \mathbf{1}} .
$$

In addition, the resulting minimum ICI power is given as $\left(\mathbf{1}^{H} \boldsymbol{\Omega}^{-1} \mathbf{1}\right)^{-1}$. It is worth noting that, in (27), there is no need to know $\beta$ or vehicular speed to obtain $\mathbf{u}^{\star}$.

\section{Method 2: ICI-Plus-Noise Power Minimization}

One may question that Method 1 does not consider noise power; thus, it may incur noise enhancement. Hence, here, we obtain the optimum combining weights that minimize the ICI-plus-noise power. To obtain the combined noise power $\mathbb{E}\left[|z(k)|^{2}\right]$, we first express noise vector $\mathbf{z}$ as

$$
\begin{aligned}
\mathbf{z} & \triangleq\left[\begin{array}{llll}
z(0) & z(1) & \cdots & z(N-1)
\end{array}\right]^{T} \\
& =\left[\begin{array}{llll}
\mathbf{e}_{0} & \mathbf{D}_{1} \mathbf{e}_{1} & \cdots & \mathbf{D}_{q} \mathbf{e}_{q}
\end{array}\right] \mathbf{u}
\end{aligned}
$$

where $\mathbf{e}_{d}$ is the frequency-domain noise vector of segment $d$, i.e.,

$$
\mathbf{e}_{d} \triangleq\left[e^{(d)}(0) \quad e^{(d)}(1) \quad \cdots \quad e^{(d)}(N-1)\right]^{T} \quad \forall d \in[0, q]
$$

$$
\begin{aligned}
& \mathbf{D}_{d} \triangleq \operatorname{diag}\left\{\left[\begin{array}{llll}
1 & \exp \left(\jmath \frac{2 \pi d}{N}\right) & \ldots & \exp \left(\jmath \frac{2 \pi(N-1) d}{N}\right)
\end{array}\right]^{T}\right\} \\
& \forall d \in[0, q]
\end{aligned}
$$

is the phase compensation matrix. Therefore, the combined noise power can be derived as

$$
\begin{aligned}
& \mathbb{E}\left[|z(k)|^{2}\right] \\
& =\frac{1}{N} \mathbb{E}\left[\mathbf{z}^{H} \mathbf{z}\right] \\
& =\frac{\mathbf{u}^{H}}{N} \mathbb{E}\left[\left(\begin{array}{cccc}
\mathbf{e}_{0}^{H} \mathbf{e}_{0} & \mathbf{e}_{0}^{H} \mathbf{D}_{1} \mathbf{e}_{1} & \ldots & \mathbf{e}_{0}^{H} \mathbf{D}_{q} \mathbf{e}_{q} \\
\vdots & \mathbf{e}_{1}^{H} \mathbf{e}_{1} & \ldots & \mathbf{e}_{1}^{H} \mathbf{D}_{q-1} \mathbf{e}_{q} \\
\vdots & \vdots & \ddots & \vdots \\
\mathbf{e}_{q}^{H} \mathbf{D}_{-q} \mathbf{e}_{0} & \mathbf{e}_{q}^{H} \mathbf{D}_{1-q} \mathbf{e}_{1} & \ldots & \mathbf{e}_{q}^{H} \mathbf{e}_{q}
\end{array}\right)\right] \mathbf{u} .
\end{aligned}
$$


In (31), the $(m, n)$ th entry of the quadratic matrix is $\mathbb{E}\left[\mathbf{e}_{m}^{H} \mathbf{D}_{n-m} \mathbf{e}_{n}\right]$. For $m=n$

$$
\mathbb{E}\left[\mathbf{e}_{m}^{H} \mathbf{e}_{m}\right]=N \sigma^{2}
$$

and for $m \neq n$

$$
\begin{aligned}
\mathbb{E}\left[\mathbf{e}_{m}^{H} \mathbf{D}_{n-m} \mathbf{e}_{n}\right] & =\mathbb{E}\left[\varepsilon_{m}^{H} \mathbf{F}^{H} \mathbf{D}_{n-m} \mathbf{F} \boldsymbol{\varepsilon}_{n}\right] \\
& =\operatorname{tr}\left(\mathbf{F}^{H} \mathbf{D}_{n-m} \mathbf{F} \mathbb{E}\left[\boldsymbol{\varepsilon}_{n} \varepsilon_{m}^{H}\right]\right) \\
& =(N-|n-m|) \sigma^{2}
\end{aligned}
$$

where $\varepsilon_{m}$ is the time-domain noise vector corresponding to segment $m$. To derive (35) from (34), we first show that, for $n>m$

$$
\begin{aligned}
\mathbb{E}\left[\boldsymbol{\varepsilon}_{n} \boldsymbol{\varepsilon}_{m}^{H}\right] & =\sigma^{2}\left(\begin{array}{cc}
\mathbf{O}_{(n-m) \times(N-n+m)} & \mathbf{O}_{(n-m) \times(n-m)} \\
\mathbf{I}_{(N-n+m)} & \mathbf{O}_{(N-n+m) \times(n-m)}
\end{array}\right) \\
& \triangleq \mathbf{E}_{n m} .
\end{aligned}
$$

Matrix $\mathbf{E}_{n m}$ is then multiplied by $\mathbf{F}^{H} \mathbf{D}_{n-m} \mathbf{F}$, and the output is the matrix with each of the entry in $\mathbf{E}_{n m}$ being upwardly circularly shifted $(n-m)$ steps, i.e., the output is

$$
\begin{aligned}
\mathbf{F}^{H} & \mathbf{D}_{n-m} \mathbf{F} \mathbf{E}_{n m} \\
& =\sigma^{2}\left(\begin{array}{cc}
\mathbf{I}_{(N-m+n)} & \mathbf{O}_{(N-m+n) \times(m-n)} \\
\mathbf{O}_{(m-n) \times(N-m+n)} & \mathbf{O}_{(m-n) \times(m-n)}
\end{array}\right) .
\end{aligned}
$$

Accordingly, (35) is verified for $n>m$. For $n<m$, (35) can be also verified in a similar way. Afterward, (31) can be expressed in a more concise form as

$$
\mathbb{E}\left[|z(k)|^{2}\right]=\mathbf{u}^{H} \mathbf{\Psi} \mathbf{u}
$$

where

$$
\boldsymbol{\Psi}=\frac{\sigma^{2}}{N}\left(\begin{array}{cccc}
N & N-1 & \cdots & N-q \\
N-1 & N & \cdots & N-q+1 \\
\vdots & \vdots & \ddots & \vdots \\
N-q & N-q+1 & \cdots & N
\end{array}\right) .
$$

Similar to Method 1, the ICI-plus-noise power minimization problem can be formulated as the following optimization problem:

$$
\begin{array}{r}
\min _{\mathbf{u} \in \mathbb{C}^{q+1}} \mathbf{u}^{H} \boldsymbol{\Gamma u} \\
\text { s.t. } \mathbf{1}^{H} \mathbf{u}=1
\end{array}
$$

where $\boldsymbol{\Gamma}=\boldsymbol{\Omega}+\boldsymbol{\Psi}$, and the optimum solution $\mathbf{u}^{\star}$ is given by

$$
\mathbf{u}^{\star}=\frac{\boldsymbol{\Gamma}^{-1} \mathbf{1}}{\mathbf{1}^{H} \boldsymbol{\Gamma}^{-1} \mathbf{1}}
$$

if $\boldsymbol{\Gamma}$ is invertible.

\section{Method 3: Interference-Plus-Noise Power Minimization Utilizing Entire Cyclic Prefix}

In the previous discussion, only the ISI-free region is utilized, and the ISI-polluted region is simply discarded. However, the
ISI-polluted region also contains signal information. Thus, it may help if we properly employ the signal segments in the ISI-polluted region. Here, we propose a method that employs the entire CP (including both ISI-polluted region and ISIfree region) and obtain the optimum combining weights that minimize the average interference-plus-noise power.

Since, in Method 3, the ISI has to be considered, we introduce index $i$ as the OFDM block index. The time-domain received signal $y_{t}\left(i\left(N+N_{g}\right)+n\right) \forall i \in \mathbb{N}$ in the ISI-polluted region, i.e., $n \in\left[-N_{g},-q\right]$, can be expressed as

$$
\begin{aligned}
y_{t}\left(i\left(N+N_{g}\right)+n\right) \\
=\sum_{l=0}^{L-1} h^{(l)}\left(i\left(N+N_{g}\right)+n\right) x_{t}^{(i)}\left((n-l)_{N}\right) \\
\quad+\varepsilon\left(i\left(N+N_{g}\right)+n\right) \\
\quad-\sum_{l=L+q+n}^{L-1} h^{(l)}\left(i\left(N+N_{g}\right)+n\right) x_{t}^{(i)}\left((n-l)_{N}\right) \\
\quad+\sum_{l=L+q+n}^{L-1} h^{(l)}\left(i\left(N+N_{g}\right)+n\right) x_{t}^{(i-1)}(n-l+N) .
\end{aligned}
$$

Without loss of generality, we consider $i=0$, and we define two functions

$\xi(n)$

$$
\triangleq \begin{cases}-\sum_{l=L+q+n}^{L-1} h^{(l)}(n) x_{t}^{(0)}\left((n-l)_{N}\right), & \text { if }-N_{g} \leqslant n<-q \\ 0, & \text { otherwise }\end{cases}
$$

$\varsigma(n)$

$$
\triangleq \begin{cases}\sum_{l=L+q+n}^{L-1} h^{(l)}(n) x_{t}^{(-1)}(n-l+N), & \text { if }-N_{g} \leqslant n<-q \\ 0, & \text { otherwise }\end{cases}
$$

to include the effects of the corresponding ICI and ISI terms caused by the signal segments in the ISI-polluted region. Hence, the received frequency-domain signal of segment $d$ on subcarrier $k$ can be expressed as

$$
\begin{aligned}
y_{F}^{(d)}(k)= & \sum_{m=0}^{N-1} x_{F}(m) H^{(d)}(k, m) e^{\frac{-\jmath 2 \pi m d}{N}}+e^{(d)}(k) \\
& +\sum_{n=0}^{N-1} \xi(n-d) e^{-\frac{j 2 \pi n k}{N}}+\sum_{n=0}^{N-1} \varsigma(n-d) e^{-\frac{j 2 \pi n k}{N}} .
\end{aligned}
$$

Similar to (15) and (17), the combined signal can be written as

$$
\begin{aligned}
v(k)= & x_{F}(k) \tilde{H}_{\mathrm{mid}}(k)+b(k)+z(k) \\
& +\sum_{d=0}^{N_{g}} u_{d} \sum_{n=0}^{N-1}(\xi(n-d)+\varsigma(n-d)) e^{-\frac{\jmath 2 \pi(n-d) k}{N}} .
\end{aligned}
$$


Compared with (17), the fourth term of (46) is the additional combined ICI-plus-ISI term, which can be written as

$\sum_{d=0}^{N_{g}} u_{d} \sum_{n=0}^{N-1}(\xi(n-d)+\varsigma(n-d)) e^{-\frac{j 2 \pi(n-d) k}{N}}=\overline{\boldsymbol{\xi}}_{k}^{H} \mathbf{u}+\overline{\boldsymbol{\varsigma}}_{k}^{H} \mathbf{u}$

where $\overline{\boldsymbol{\xi}}_{k}$ and $\overline{\boldsymbol{\zeta}}_{k}$ are defined as

$\overline{\boldsymbol{\xi}}_{k} \triangleq\left[\begin{array}{lll}\sum_{n=0}^{N-1} \xi(n) e^{-\frac{j 2 \pi n k}{N}} & \cdots & \sum_{n=0}^{N-1} \xi\left(n-N_{g}\right) e^{-\frac{j 2 \pi\left(n-N_{g}\right) k}{N}}\end{array}\right]^{H}$

$\overline{\boldsymbol{\varsigma}}_{k} \triangleq\left[\begin{array}{lll}\sum_{n=0}^{N-1} \varsigma(n) e^{-\frac{j 2 \pi n k}{N}} & \cdots & \sum_{n=0}^{N-1} \varsigma\left(n-N_{g}\right) e^{-\frac{j 2 \pi\left(n-N_{g}\right) k}{N}}\end{array}\right]^{H}$

respectively. For brevity, we define

$$
\Upsilon \triangleq \frac{1}{N} \mathbb{E}\left[\overline{\boldsymbol{\xi}}_{k} \overline{\boldsymbol{\xi}}_{k}^{H}\right] \text {. }
$$

It is shown in Appendix A that the $(r, s)$ th entry of matrix $\Upsilon$ is given as

$$
\begin{cases}\frac{1}{N} \sum_{n=-s}^{N-1-r}\left(\sum_{l=L+q+n}^{L-1} \Xi_{l}\right), & \text { if } r-s \geqslant 0 \\ \frac{1}{N} \sum_{n=-r}^{N-1-s}\left(\sum_{l=L+q+n}^{L-1} \Xi_{l}\right), & \text { if } r-s<0\end{cases}
$$

and $\mathbb{E}\left[\overline{\boldsymbol{\zeta}}_{k} \overline{\boldsymbol{\varsigma}}_{k}^{H}\right]=\mathbb{E}\left[\overline{\boldsymbol{\xi}}_{k} \overline{\boldsymbol{\xi}}_{k}^{H}\right]=\boldsymbol{\Upsilon}$. Therefore, the interference-plusnoise power can be written as $\mathbf{u}^{H}(\boldsymbol{\Omega}+\boldsymbol{\Psi}+2 \boldsymbol{\Upsilon}) \mathbf{u}$, where $\boldsymbol{\Omega}$ and $\Psi$ are the same as those in (25) and (40), except that the dimensions here are $\left(N_{g}+1\right) \times\left(N_{g}+1\right)$ instead of $(q+1) \times$ $(q+1)$.

Similar to Methods 1 and 2, the interference-plus-noise power minimization problem can be expressed as the following optimization problem:

$$
\begin{aligned}
& \min _{\mathbf{u} \in \mathbb{C}^{N_{g}+1}} \mathbf{u}^{H}(\boldsymbol{\Omega}+\boldsymbol{\Psi}+2 \boldsymbol{\Upsilon}) \mathbf{u} \\
& \text { s.t. } \quad \mathbf{1}^{H} \mathbf{u}=1 .
\end{aligned}
$$

Similar to (27) and (41), the global optimum solution $\mathbf{u}^{\star}$ is given by

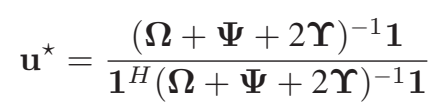

if $(\boldsymbol{\Omega}+\boldsymbol{\Psi}+2 \boldsymbol{\Upsilon})$ is invertible.

\section{COMPLEXITy COMPARISON}

\section{A. Calculation of the Combining Weights}

Table I demonstrates the required knowledge to calculate the combining weights for the proposed methods. We observe that Method 1 is the simplest method, which requires only the knowledge of the ISI-free region. The ISI-free region can be detected in the synchronization stage using traditional methods
TABLE I

Required KNowledge of the Three Methods

\begin{tabular}{|c|c|c|}
\hline Method 1 & Method 2 & Method 3 \\
\hline \hline \multirow{3}{*}{ ISI-free region } & ISI-free region & Power delay profile \\
& Vehicular speed & Vehicular speed \\
& Noise power & Noise power \\
\hline
\end{tabular}

such as in [32] and [33]. For Method 2, we should know both vehicular speed (or, equivalently, $\beta$ ) and noise power in advance. Among the three methods we propose, Method 3 is the most complicated one, for it requires the additional knowledge of the PDP instead of the ISI-free region.

To calculate the combining weights, a matrix inversion is required in general. However, for Method 1, it is suggested by curve fitting to our numerical results that the optimum combining weights $\mathbf{u}^{\star}$ can be explicitly represented as

$$
\mathbf{u}^{\star}=\frac{1}{N}\left[\frac{1}{2}(N-q+1) \quad \mathbf{1}^{T} \quad \frac{1}{2}(N-q+1)\right]^{T} .
$$

The optimality of expression (54) is proven in Appendix B. Hence, for Method 1, the matrix inversion can be totally avoided, and its complexity is extremely low. As for Method 2, since $\boldsymbol{\Gamma}$ is a symmetric positive definite Toeplitz matrix, we can use the Levinson algorithm to evaluate $\Gamma^{-1} 1$, which requires $4 q^{2}$ flops (a flop denotes any arithmetic operation, such as a multiplication or an addition operation) in general [36]. For Method 3, the optimum combining weight $\mathbf{u}^{\star}$ can be evaluated by the Gaussian elimination method, which in general requires $2 N_{g}^{3} / 3$ flops [36]. In summary, the complexity values to compute the combining weights is $\mathcal{O}(1), \mathcal{O}\left(q^{2}\right)$, and $\mathcal{O}\left(N_{g}^{3}\right)$ for Methods 1, 2, and 3, respectively.

It is worth mentioning that, since all the parameters required for the calculation of the combining weights vary slowly compared with the symbol rate, the combining weights do not require to be calculated frequently.

\section{B. Low-Complexity Implementation of the OFDM Segment Combining}

For convenience, we denote $g$ as the number of the combining weights, namely, $g=q$ for Methods 1 and 2, and $g=N_{g}$ for Method 3. The relationship between frequencydomain combined signal $v(k)$ and received OFDM segments is expressed as (15). However, it is too complicated to implement segment combining suggested by (15) directly, i.e., transforming each time-domain segment into a frequency-domain segment and letting each frequency-domain segment be weighted and added since it requires $g$ times of FFT operations and many other arithmetic operations. From (4) and [15], the combined frequency-domain signal $v(k)$ can be rewritten as

$$
\begin{aligned}
v(k) & =\sum_{n=0}^{N-1} \sum_{d=0}^{g} u_{d}\left[\mathbf{y}_{t}^{(d)}\right]_{n} e^{\frac{j 2 \pi k d}{N}} e^{\frac{-\jmath 2 \pi n k}{N}} \\
& =\sum_{n=0}^{N-1} \sum_{d=0}^{g} u_{d} y_{t}(n-d) e^{\frac{-\jmath 2 \pi k(n-d)}{N}} .
\end{aligned}
$$




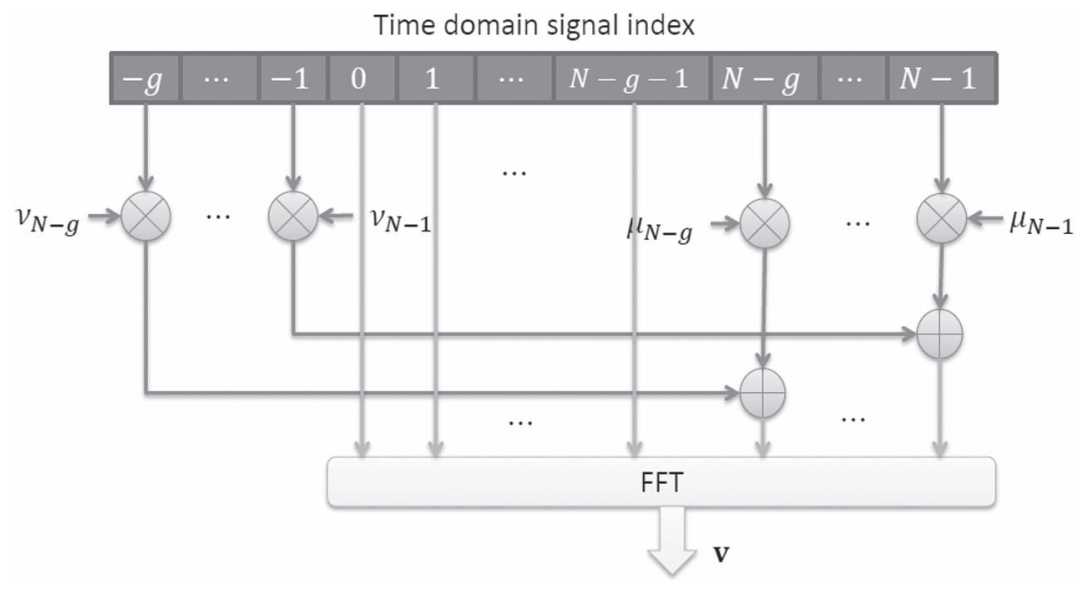

Fig. 3. Low-complexity implementation of segment combining.

After changing the variables and some mathematic manipulations, (55) can be expressed as

$$
\begin{aligned}
v(k)= & \sum_{n=0}^{N-g-1} y_{t}(n) e^{\frac{-\jmath 2 \pi n k}{N}} \\
& +\sum_{n=N-g}^{N-1}\left(\mu_{n} y_{t}(n)+\nu_{n} y_{t}(n-N)\right) e^{\frac{-\jmath 2 \pi n k}{N}}
\end{aligned}
$$

where $\mu_{n}=\sum_{d=0}^{N-1-n} u_{d}$, and $\nu_{n}=1-\mu_{n}$. As suggested by (56), segment combining can be simply implemented by the circuit in Fig. 3. Note that the implementation requires only one instead of $g$ times of FFT operations, as compared with the direct implementation through (15), and the number of extra multiplications and additions is greatly reduced. In general, the segment combining through the implementation in Fig. 3 requires $4 g$ real multiplications and $g$ complex additions.

\section{Numerical Results}

\section{A. Channel Estimation and Equalization}

Here, we introduce the channel estimator and equalizer used in our simulations. We assume that pilot symbols are placed on subcarriers whose indexes are given in set $\mathcal{P}$, and we use $\left[\mathbf{x}_{F}\right]_{\mathcal{P}}$ and $[\mathbf{v}]_{\mathcal{P}}$ to denote the pilot symbol vector and combined signal vector on the corresponding subcarriers. We rewrite the combined samples $\{v(k)\}_{k=0}^{N-1}$ in a vector form as

$$
\mathbf{v} \triangleq\left[\begin{array}{llll}
v(0) & v(1) & \cdots & v(N-1)
\end{array}\right]^{T} .
$$

By (19), the combined signal vector in the index set $\mathcal{P}$ can be rewritten as

$$
[\mathbf{v}]_{\mathcal{P}}=\mathbf{X}_{\mathcal{P}}[\mathbf{F}]_{(\mathcal{P}, \mathcal{L})} \tilde{\mathbf{h}}_{\text {mid }}+[\tilde{\mathbf{z}}]_{\mathcal{P}}
$$

where

$$
\mathbf{X}_{\mathcal{P}}=\operatorname{diag}\left\{\left[\mathbf{x}_{F}\right]_{\mathcal{P}}\right\}
$$

$\tilde{\mathbf{h}}_{\text {mid }}$ is the equivalent time-domain channel vector corresponding to the path-delay index set $\mathcal{L}$ after the segment combination,
TABLE II

SimUlATION PARAMETERS

\begin{tabular}{|c||c|}
\hline Bandwidth & $8 \mathrm{MHz}$ \\
\hline FFT Size & 2048 \\
\hline Modulation & QPSK \\
\hline Carrier Frequency & $700 \mathrm{MHz}$ \\
\hline Number of Pilots & 45 \\
\hline Normalized Doppler Frequency $f_{D}$ & 0.05 \\
\hline Corresponding Vehicle Speed & $300(\mathrm{~km} / \mathrm{hr})$ \\
\hline
\end{tabular}

and $\tilde{\mathbf{z}}$ is the interference-plus-noise term. Hence, by using the LS criterion, $\tilde{\mathbf{h}}_{\text {mid }}$ can be estimated as [26], [27]

$$
\left([\mathbf{F}]_{(\mathcal{P}, \mathcal{L})}^{H} \mathbf{X}_{\mathcal{P}}^{H} \mathbf{X}_{\mathcal{P}}[\mathbf{F}]_{(\mathcal{P}, \mathcal{L})}\right)^{-1}[\mathbf{F}]_{(\mathcal{P}, \mathcal{L})}^{H} \mathbf{X}_{\mathcal{P}}^{H}[\mathbf{v}]_{\mathcal{P}}
$$

To derive a frequency-domain one-tap equalizer, (19) can be rewritten as

$$
\mathbf{v}=\tilde{\mathbf{H}}_{\text {mid }} \mathbf{x}_{F}+\tilde{\mathbf{z}}
$$

where $\tilde{\mathbf{H}}_{\text {mid }} \triangleq \operatorname{diag}\left\{[\mathbf{F}]_{(\mathcal{S}, \mathcal{L})} \tilde{\mathbf{h}}_{\text {mid }}\right\}$, and $\mathcal{S} \triangleq\{0,1, \ldots, N-$ $1\}$. A one-tap equalizer simply compensates for the distortion caused by the channel frequency response and can be mathematically described as

$$
\hat{\mathbf{x}}_{F}=\tilde{\mathbf{H}}_{\text {mid }}^{-1} \mathbf{v} .
$$

Then, data are detected directly.

\section{B. Simulation Environment}

The system parameters used for the computer simulations are listed in Table II. The OFDM frame structure follows the $2 \mathrm{~K}$ mode in DVB-H specification [38], unless otherwise stated. The time-varying channel is simulated according to Jakes' model [35]. The multipath PDPs follow ITU Veh. A channel and ITU Veh. B channel [39], whose maximum path delay $L-1$ equals to 21 and 161 , separately.

\section{Simulation Results}

In Fig. 4, we show the ICI suppression capabilities of Method 1, the methods in [20] (denoted as the Chang method), 


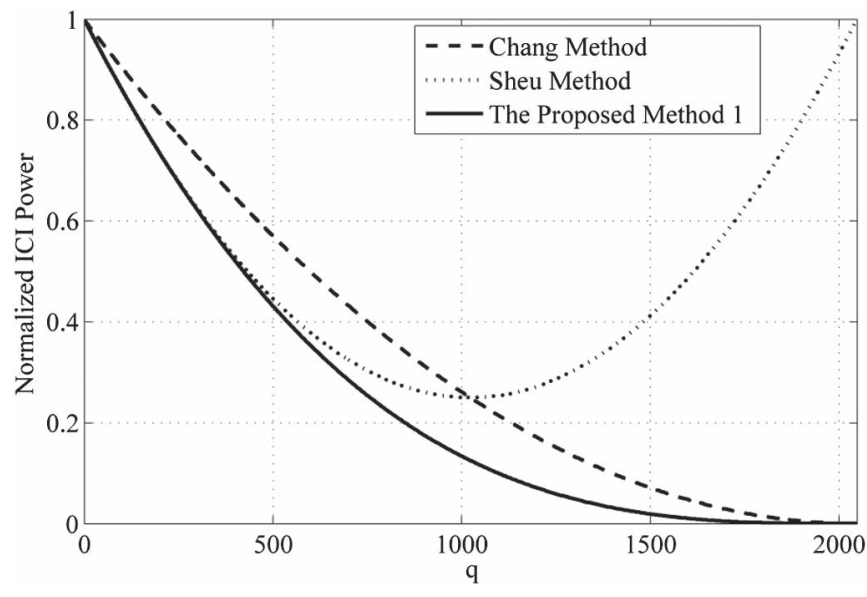

Fig. 4. ICI suppression capability comparison of Method 1 and the methods in [20] and [21] as a function of $q$.

and [21] (denoted as the Sheu method), as a function of $q$. The metric normalized ICI power represents the ratio of the ICI power after segment combining to that before segment combining, and it is mathematically defined as

$$
\text { Normalized ICI Power }(\mathbf{u}) \triangleq \frac{\mathbf{u}^{T} \boldsymbol{\Omega} \mathbf{u}}{\mathbf{i}^{T} \boldsymbol{\Omega i}}
$$

where $\mathbf{i} \triangleq[1,0, \ldots, 0]^{T}$. The denominator in (63) is the ICI power before segment combining.

As expected, it is apparent that Method 1 is always better than or as good as the Sheu and Chang methods. We also observe an interesting fact that the larger $q$ is, the better the Chang method is, and the worse performance the Sheu method exhibits, and vice versa. This phenomenon can be explained as follows. Since the combining weights of the Chang method converge to that of Method 1, which is the optimum solution, as $q$ approaches to $N-1$, the better the Chang method will perform accordingly. On the other hand, the combining weights of the Sheu method become similar to that of Method 1 as $q$ approaches to 1; therefore, the Sheu method will be better also when $q$ is closer to 1 . In addition, it is worth noting that, when $q=2047$, the normalized ICI power equals to zero for both Method 1 and Chang method. This means that the ICI effect is completely removed, and this result agrees with the conclusion drawn in [20].

In Fig. 5, the BER performance of Method 1 in ITU Veh. A channel is demonstrated. Although not specified in the DVB standard, we artificially extend the CP length to simulate the $\mathrm{CP}=N / 2$ and $\mathrm{CP}=N$ cases to make the simulation results complete. Because these two cases are not supported by real DVB systems, they are plotted in dashed lines. It is obvious that Method 1 outperforms the original one-tap equalizer even for the most modest $\mathrm{CP}$ length, namely, $N / 32$. At the BER level of $3 \times 10^{-3}$, Method 1 provides about 1 - and 5 -dB improvements over the original one-tap equalizer when the length of the $\mathrm{CP}$ is $N / 32$ and $N / 4$, respectively. The performance improvement is remarkable if we consider the extremely low complexity Method 1 has. As expected, as the length of the $\mathrm{CP}$ increases, the ICI suppression capability increases as well. When the CP length equals to $N$, the performance of Method 1

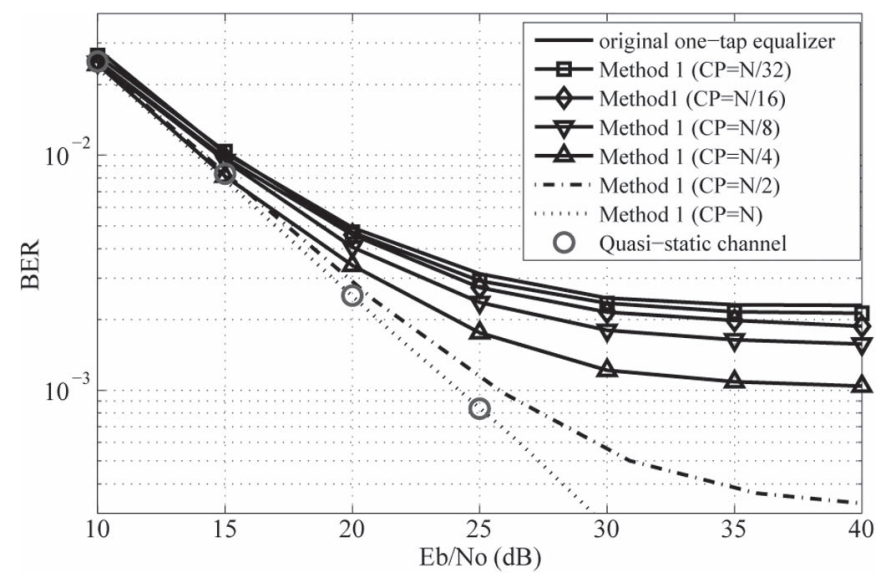

Fig. 5. BER performance of Method 1 with different CP length in the ITU Veh. A channel.

is indistinguishable from that of a quasi-static channel. This is because, for this case, the ICI effect is almost canceled, as shown in Fig. 4 and [20].

In Figs. 6 and 7, we show the BER performances of the proposed methods in ITU Veh. A channel and Veh. B channel, respectively. The $\mathrm{CP}$ length is set as $N / 8$. Because the performance curves of the three proposed methods in Fig. 6(a) are hardly distinguishable, a zoom-in section is given in Fig. 6(b). From these figures, we observe the following two facts: 1) the performance of Method 1 is almost identical to that of Method 2; and 2) Method 3 outperforms the other two methods, but the performance gap is more evident in the Veh. B channel than in the Veh. A channel.

The first observation seems to be contradictory to the conventional engineering intuition. Since Method 2 takes noise effect into consideration, it should outperform Method 1, particularly in the noise-dominated region. The key reason for this result is that the noise-suppression capabilities of the two methods are similar. For brevity, the detailed explanation is given in Appendix C.

For explaining the second observation, we note that, by exploiting the ISI-polluted region, we can use more signal segments for ICI mitigation, and this is the main reason that Method 3 outperforms the other two methods. In the Veh. A channel, since the delay spread is very small, which makes the ISI-polluted region small as well, the performance improvement over the other two methods is insignificant. On the contrary, the delay spread of the Veh. B channel is larger so that the advantage of Method 3 clearly exhibits.

In conclusion, we suggest from our simulation results that we should use Method 1 if channel delay spread is small and use Method 3 when channel delay spread is large. On the other hand, because Methods 1 and 2 are indistinguishable in BER performance, it is suggested not to use Method 2, which has much higher computational complexity.

Fig. 8(a) and (b) shows the performance of the proposed Method 1 as a function of normalized Doppler frequency with $E_{b} / N_{o}$ being equal to $20 \mathrm{~dB}$, adopting quadrature phase-shift keying (QPSK) and 16-ary quadrature amplitude modulation (16QAM), respectively. It is revealed in Fig. 8 that the proposed method is robust in the following two aspects. First, the 


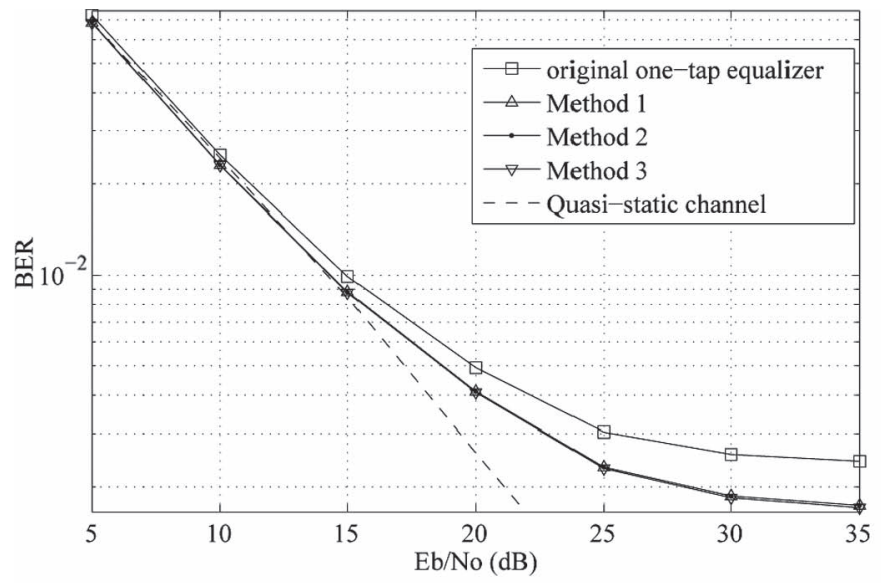

(a)

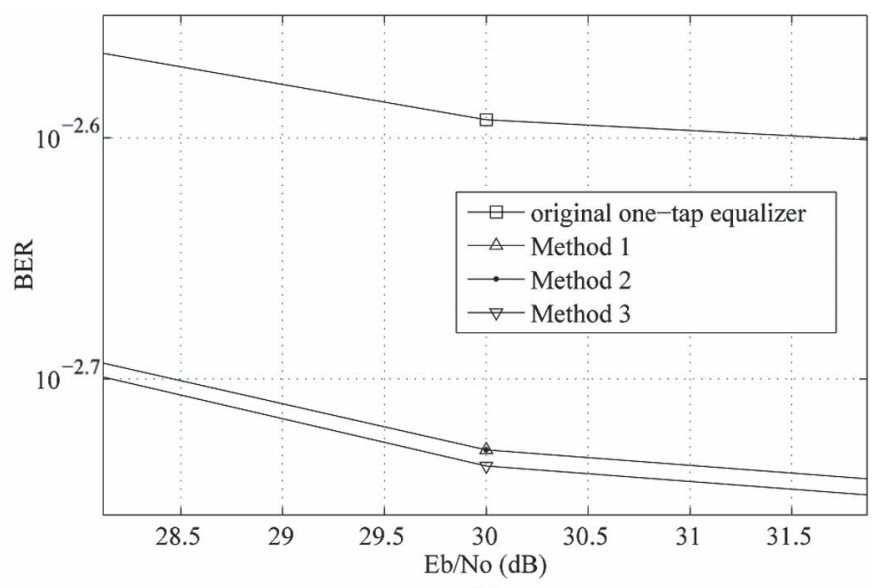

(b)

Fig. 6. BER performance of the proposed methods in the ITU Veh. A channel. (a) BER performance of the proposed methods. (b) Zoom-in section.

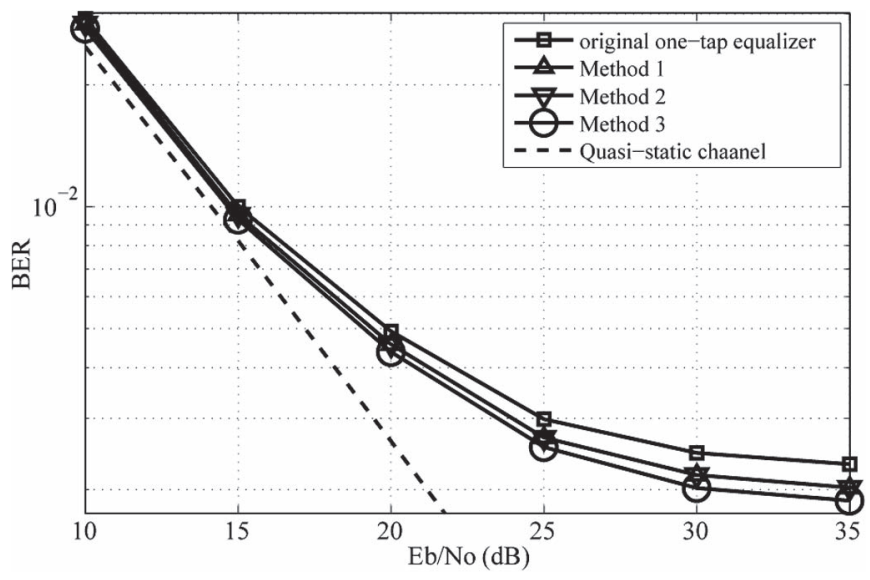

Fig. 7. BER performance of the proposed methods in the ITU Veh. B channel.

proposed method is robust in extremely high mobility environments. That is, even when the vehicular speed is unreasonably high that linear approximation on channel variation (6) is no longer valid, e.g., $f_{D}=0.3$ (the vehicular speed is $1800 \mathrm{~km} / \mathrm{h}$ according to our simulation parameters), the proposed method still improves the BER performance. Second, the proposed method is robust against nonideal ISI-free region detection. We examine the effect caused by nonideal ISI-free region detection by simulating Method 1 in conjunction with the conventional ISI-free region detection method [33] ${ }^{1}$ in Fig. 8. In Fig. 9, we execute a simulation to show the probability mass function (PMF) of the detection error of the method [33], where $\eta$ represents the detection error, which is defined as $\eta=\hat{q}-q$, and $\hat{q}$ denotes the detected output of the method [33]. In Fig. 9, we observe that the detection error is nonnegligible. Although the ISI-free region detection method [33] has notable detection error, it is shown in Fig. 8(a) and (b) that the performance loss is negligible. Hence, we conclude that the proposed method is a robust method for practical use.

\footnotetext{
${ }^{1}$ We assume that five randomly generated OFDM symbols are used for ISIfree region detection.
}

\section{CONCLUSION}

In this paper, three sets of segment combining weights have been optimally derived in different aspects for OFDM systems in high-mobility multipath fading channels. Compared with other ICI suppression methods using segment combining [20][23], Method 1 achieves a better performance with the same computation complexity. The performance can be improved by Method 3 if the delay spread is large. The proposed methods also have the following advantages. First, the proposed methods have very low complexity, as shown in Section IV. Second, they are highly compatible with most existing data detection, equalization, and channel estimation methods. Third, although the derivations in this paper are based on OFDM signals, the methods can be directly applied to other CP communication systems, e.g., orthogonal frequency-division multiple access, single-carrier block transmission, single-carrier frequency-division multiple access and CP-aided code-division multiple access.

\section{APPENDIX A}

$$
\text { DeRIVATION OF (51) AND } \mathbb{E}\left[\overline{\boldsymbol{\zeta}}_{k} \overline{\boldsymbol{\zeta}}_{k}^{H}\right]=\mathbb{E}\left[\overline{\boldsymbol{\xi}}_{k} \overline{\boldsymbol{\xi}}_{k}^{H}\right]=\boldsymbol{\Upsilon}
$$

The additional average ICI power after segment combining is given as

$$
\frac{1}{N} \mathbf{u}^{H} \mathbb{E}\left[\overline{\boldsymbol{\xi}}_{k} \overline{\boldsymbol{\xi}}_{k}^{H}\right] \mathbf{u}=\mathbf{u}^{H} \boldsymbol{\Upsilon} \mathbf{u}
$$

The $(r, s)$ th entry of matrix $\Upsilon \mathbf{\Upsilon}=\mathbb{E}\left[\overline{\boldsymbol{\xi}}_{k} \overline{\boldsymbol{\xi}}_{k}^{H}\right] / N$ can be expressed as

$$
\begin{gathered}
\left.\frac{1}{N} \mathbb{E}\left[\overline{\boldsymbol{\xi}}_{k}\right]_{r}\left(\left[\overline{\boldsymbol{\xi}}_{k}\right]_{s}\right)^{*}\right]=\frac{1}{N} \sum_{n=0}^{N-1} \sum_{m=0}^{N-1} \mathbb{E}\left[\xi(n-r) \xi^{*}(m-s)\right] \\
\cdot \exp \left\{\frac{j 2 \pi(-n k+k r+m k-k s)}{N}\right\}
\end{gathered}
$$

Since $\left.\mathbb{E}\left[\overline{\boldsymbol{\xi}}_{k}\right]_{r}\left(\left[\overline{\boldsymbol{\xi}}_{k}\right]_{s}\right)^{*}\right]=0$ for $n-r \geqslant-q$ or $m-s \geqslant-q$, we only consider the case $-N_{g} \leqslant n-r<-q$ and $-N_{g} \leqslant m-s<-q$. 


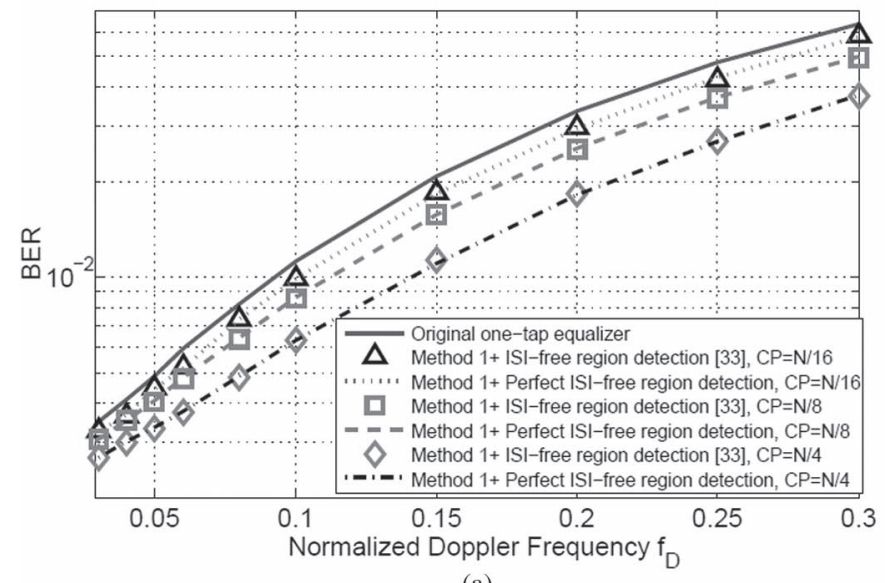

(a)

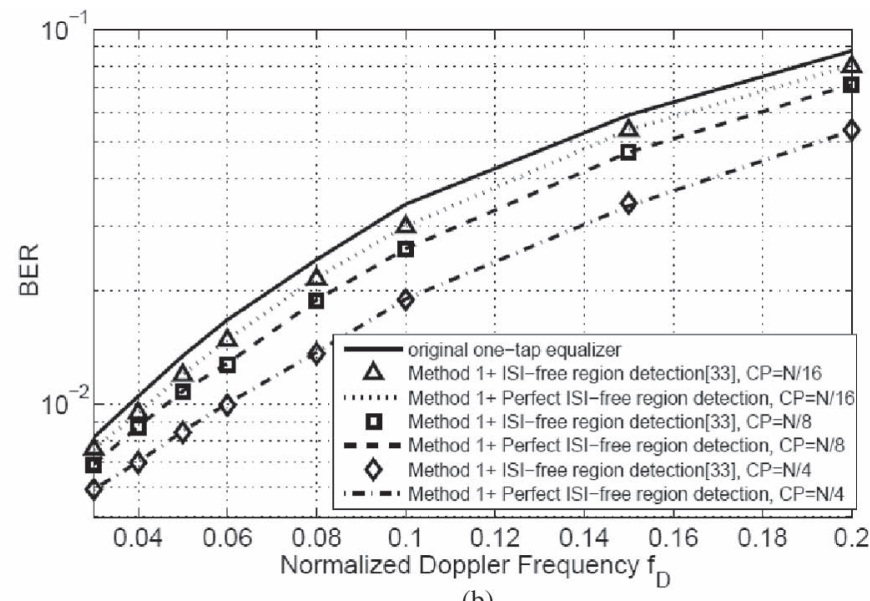

(b)

Fig. 8. BER performance of Method 1 as a function of $f_{D}$ in the ITU Veh. A channel $\left(E_{b} / N_{o}=20 \mathrm{~dB}\right)$. (a) QPSK. (b) 16QAM.

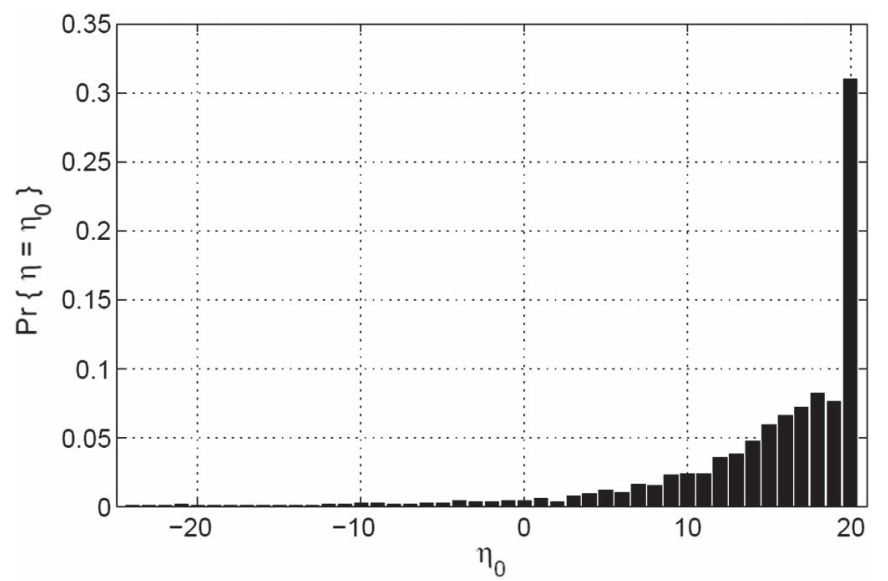

Fig. 9. PMF of the detection error of the ISI-free region detection method in [33] $\left(f_{D}=0.1, E_{b} / N_{o}=20 \mathrm{~dB}\right.$, and QPSK modulation).

In this region

$$
\begin{aligned}
\frac{1}{N} \mathbb{E} & {\left[\xi(n-r) \xi^{*}(m-s)\right] } \\
= & \frac{1}{N} \sum_{l_{1}=L+q+n-r}^{L-1} \sum_{l_{2}=L+q+m-s}^{L-1} \mathbb{E}\left[h^{\left(l_{1}\right)}(n-r) h^{*\left(l_{2}\right)}(m-s)\right] \\
& \cdot \mathbb{E}\left[x_{t}^{(i)}\left(\left(n-r-l_{1}\right)_{N}\right) x_{t}^{*(i)}\left(\left(m-s-l_{2}\right)_{N}\right)\right] .
\end{aligned}
$$

We assume that the channels are uncorrelated scattering and the transmit data signals are independent and identically distributed; it is directly implied by these two assumptions that $\mathbb{E}\left[h^{\left(l_{1}\right)}(m) h^{*\left(l_{2}\right)}(n)\right]=0$ if $l_{1} \neq l_{2}, \forall m, n \in \mathbb{Z}$ and $\mathbb{E}\left[x_{t}^{(i)}(m)\right.$ $\left.x_{t}^{*(i)}(n)\right]=\delta(m-n), \forall m, n \in[0, N-1]$, where $\delta(\cdot)$ is the Kronecker delta function defined as

$$
\delta(x) \triangleq \begin{cases}1, & \text { if } x=0 \\ 0, & \text { otherwise. }\end{cases}
$$

Hence, (66) can be further derived as

$$
\begin{aligned}
& \frac{1}{N} \mathbb{E}\left[\xi(n-r) \xi^{*}(m-s)\right] \\
& \quad=\frac{1}{N} \sum_{l=L+q+\max \{n-r, m-s\}}^{L-1} \Xi_{l} \delta(n-r-m+s) .
\end{aligned}
$$

In general

$$
\begin{aligned}
& \mathbb{E}\left[\xi(n-r) \xi^{*}(m-s)\right] \\
& \quad= \begin{cases}\sum_{l=L+q+n-r}^{L-1} \Xi_{l}, & \text { if }-N_{g} \leqslant n-r=m-s<-q \\
0, & \text { otherwise. }\end{cases}
\end{aligned}
$$

Therefore, (65) can be formulated as

$$
\begin{aligned}
\left.\frac{1}{N} \mathbb{E}\left[\overline{\boldsymbol{\xi}}_{k}\right]_{r}\left(\left[\overline{\boldsymbol{\xi}}_{k}\right]_{s}\right)^{*}\right] \\
\quad=\frac{1}{N} \sum_{n=0}^{N-1} \sum_{m=0}^{N-1} \delta(n-r-m+s) \sum_{l=L+q+n-r}^{L-1} \Xi_{l} \\
\quad= \begin{cases}\frac{1}{N} \sum_{n=-s}^{N-1-r}\left(\sum_{l=L+q+n}^{L-1} \Xi_{l}\right), & \text { if } r-s \geqslant 0 \\
\frac{1}{N} \sum_{n=-r}^{N-1-s}\left(\sum_{l=L+q+n}^{L-1} \Xi_{l}\right), & \text { if } r-s<0 .\end{cases}
\end{aligned}
$$

On the other hand, the combined ISI power is given by

$$
\frac{1}{N} \mathbf{u}^{H} \mathbb{E}\left[\overline{\boldsymbol{\zeta}}_{k} \overline{\boldsymbol{\zeta}}_{k}^{H}\right] \mathbf{u} .
$$

The $(r, s)$ th entry of matrix $\mathbb{E}\left[\overline{\boldsymbol{\zeta}}_{k} \overline{\boldsymbol{\zeta}}_{k}^{H}\right] / N$ equals to

$$
\begin{aligned}
\frac{1}{N} \sum_{n=0}^{N-1} \sum_{m=0}^{N-1} \mathbb{E}\left[\varsigma(n-r) \varsigma^{*}(m-s)\right] \\
\cdot \exp \left\{\frac{\jmath 2 \pi(-n k+k r+m k-k s)}{N}\right\} .
\end{aligned}
$$

Similarly, for $-N_{g} \leqslant n<-q$

$$
\begin{aligned}
\frac{1}{N} \mathbb{E} & {\left[\varsigma(n-r) \varsigma^{*}(m-s)\right] } \\
= & \frac{1}{N} \sum_{l_{1}=L+q+n-r l_{2}=L+q+m-s}^{L-1} \sum_{\left.\left(n-r-l_{1}+N\right) x_{t}^{*(i-1)}\left(m-s-l_{2}+N\right)\right]}^{L-1} \mathbb{E}\left[h^{\left(l_{1}\right)}(n-r) h^{*\left(l_{2}\right)}(m-s)\right] \\
& \cdot \mathbb{E}\left[x_{t}^{(i-1)}(n-r)\right. \\
= & \frac{1}{N} \sum_{l=L+q+\max \{n-r, m-s\}}^{L-1} \Xi_{l} \delta(n-r-m+s) .
\end{aligned}
$$


Hence, the $(r, s)$ th entry of $\mathbb{E}\left[\overline{\boldsymbol{\zeta}}_{k} \overline{\boldsymbol{\zeta}}_{k}^{H}\right] / N$ equals to

$$
\left\{\begin{array}{cl}
\frac{1}{N} \sum_{n=-s}^{N-1-r}\left(\sum_{l=L+q+n}^{L-1} \Xi_{l}\right), & \text { if } r-s \geqslant 0 \\
\frac{1}{N} \sum_{n=-r}^{N-1-s}\left(\sum_{l=L+q+n}^{L-1} \Xi_{l}\right), & \text { if } r-s<0 .
\end{array}\right.
$$

Accidentally, (74) is identical to (70); hence, we can conclude that $\mathbb{E}\left[\overline{\boldsymbol{\zeta}}_{k} \overline{\boldsymbol{\varsigma}}_{k}^{H}\right]=\mathbb{E}\left[\overline{\boldsymbol{\xi}}_{k} \overline{\boldsymbol{\xi}}_{k}^{H}\right]=\Upsilon$.

\section{APPENDIX B}

\section{VERIFICATION OF THE OPTIMALITY OF THE EXPLICIT EXPRESSION OF $\mathbf{u}^{\star}(54)$ FOR METHOD 1}

We will show that the explicit expression (54) is the global minimizer for the optimization problem (25) by showing that the KKT condition (26a), as well as (26b), is satisfied. At first, it is obvious that (26b) is satisfied. In the following, we will show that (26a) is satisfied as well. From (24), the covariance matrix $\Omega$ can be explicitly expressed as

$$
\begin{aligned}
\boldsymbol{\Omega}=\kappa_{1} & \sum_{n=1}^{N-1}|\Phi(n)|^{2} \\
& \times\left(\begin{array}{cccc}
1 & e^{\frac{-\jmath 2 \pi n}{N}} & \cdots & e^{\frac{-\jmath 2 \pi n q}{N}} \\
e^{\frac{-\jmath 2 \pi n}{N}} & 1 & \cdots & e^{\frac{-\jmath 2 \pi n(q-1)}{N}} \\
\vdots & & \ddots & \vdots \\
e^{\frac{-\jmath 2 \pi n q}{N}} & e^{\frac{-\jmath 2 \pi n(q-1)}{N}} & \cdots & 1
\end{array}\right)
\end{aligned}
$$

where $\kappa_{1}$ is a constant. For convenience, we define $\Phi(0) \triangleq 0$. Then, (75) can be expressed as

$$
\kappa_{1}\left(\begin{array}{cccc}
\Phi_{F}(0) & \Phi_{F}(1) & \cdots & \Phi_{F}(q) \\
\Phi_{F}(1) & \Phi_{F}(0) & \cdots & \Phi_{F}(q-1) \\
\vdots & \vdots & \ddots & \vdots \\
\Phi_{F}(q) & \Phi_{F}(q-1) & \cdots & \Phi_{F}(0)
\end{array}\right)
$$

where $\left\{\Phi_{F}(k)\right\}_{k=0}^{N-1}$ is the DFT of the sequence $\left\{|\Phi(n)|^{2}\right\}_{n=0}^{N-1}$. We apply (54) into the left-hand side of (26a), namely, $\Omega \mathbf{u}$, and its $k$ th entry is obtained by

$$
\kappa_{2}\left(\frac{(N-q+1)}{2}\left[\Phi_{F}(N-k)+\Phi_{F}(q-k)\right]-\sum_{d=q}^{N} \Phi_{F}(d-k)\right)
$$

where $\kappa_{2}$ is a constant. Since $|\Phi(n)|^{2}$ is a real and symmetric function, its DFT can be expressed as

$$
\Phi_{F}(k)=2 \sum_{n=1}^{N / 2-1}|\Phi(n)|^{2} \cos \left(\frac{2 \pi n k}{N}\right)
$$

The term $\cos (2 \pi n k / N)$ with small $n$ and $k$ can be approximated as (by Taylor series expansion)

$$
\cos \left(\frac{2 \pi n k}{N}\right) \approx 1-\frac{1}{2}\left(\frac{2 \pi n k}{N}\right)^{2}
$$

Since $|\Phi(n)|^{2}$ is a nearly band-limited function, only the terms close to the origin have a significant impact on the final result. Therefore, (78) can be approximated as

$\Phi_{F}(k) \approx 2 \sum_{n=1}^{N / 2-1}|\Phi(n)|^{2}\left(1-\frac{1}{2}\left(\frac{2 \pi n k}{N}\right)^{2}\right), k=0, \ldots, \frac{N}{2}-1$.

After change of variable,

$\Phi_{F}\left(k^{\prime}\right) \approx \kappa_{3}\left(k^{\prime}-\frac{N}{2}\right)^{2}+$ const $, \quad k^{\prime}=0,1, \ldots, N-1$

where $\kappa_{3}$ is a constant. Applying (81) to (77), we can deduce that all the entries of $\Omega \mathbf{u}^{\star}$ are equal to each other; as a result, the equality (26a) is verified. Therefore, we can conclude that $\mathbf{u}^{\star}$ suggested in (54) is very close to the global optimum solution for Method 1. We made a numerical exhaustive search experiment, for which FFT size, or $N$, is varied from 16 to 4096 and $q$ ranges from 1 to $N-1$. In the numerical experiment, we do not see any meaningful difference between the exact optimum global optimizer (27) and our proposed solution (54). Therefore, we can claim that the approximation is very accurate, and solution (54) is optimum at least for $N \leqslant 4096$.

In [24], the authors claimed that the combining coefficients are optimum for ICI minimization when there is no timedomain channel variations in the sample region $[N-q, N-$ 1], and they showed that the Frank function window combining coefficients satisfy the criterion. However, the authors did not give a formal proof in [24]. Now, the given claim has been proven to be valid since the method in [24] is equivalent to Method 1, which can be easily verified by applying (54)-(56).

\section{APPENDIX C}

\section{Explanation of the Fact: The Performances of Methods 1 AND 2 ARE Almost IDENTICAL}

At first, we consider the combining weights that minimize the noise power, i.e., the following optimization problem:

$$
\begin{array}{r}
\min _{\mathbf{u} \in \mathbb{C}^{q+1}} \mathbf{u}^{H} \boldsymbol{\Psi} \mathbf{u} \\
\text { s.t. } \mathbf{1}^{H} \mathbf{u}=1 .
\end{array}
$$

The KKT condition for problem (82) is

$$
\mathbf{\Psi} \mathbf{u}=\lambda \mathbf{1} \quad \text { and } \quad \mathbf{1}^{T} \mathbf{u}=1
$$

The solution

$$
\mathbf{u}_{\mathrm{NOISE}}^{\star}=\left[\begin{array}{lll}
\frac{1}{2} & \mathbf{0}^{T} & \frac{1}{2}
\end{array}\right]^{T}
$$

satisfies the KKT condition (83); therefore, it is a global minimizer. It is easy to derive the noise power caused by $\mathbf{u}_{\text {NOISE }}^{\star}$ as

$$
\frac{1}{N} \mathbf{u}_{\text {NOISE }}^{\star T} \boldsymbol{\Psi} \mathbf{u}_{\text {NOISE }}^{\star}=\left(1-\frac{q}{2 N}\right) \sigma^{2} .
$$


On the other hand, by straightforward calculations, the corresponding noise power of the proposed Method 1 can be derived as

$$
\left(1-\frac{q}{2 N}\right) \sigma^{2}+\frac{q\left(q^{2}-1\right)}{6 N^{3}} \sigma^{2} \approx\left(1-\frac{q}{2 N}\right) \sigma^{2} .
$$

The approximation in (86) is made due to the fact that $q \ll N$ in practice. It is observed that (86) is identical to (85), which means that the noise suppression capability of Method 1 is almost optimum. Since Method 1 almost minimizes the noise power, Method 2 cannot obviously outperform Method 1 in its noise suppression capability. Therefore, the performance of Method 1 is indistinguishable from that of Method 2.

\section{REFERENCES}

[1] M. Russell and G. L. Stüber, "Interchannel interference analysis of OFDM in a mobile environment," in Proc. IEEE Veh. Technol. Conf., Jul. 1995, vol. 2, pp. 820-824.

[2] G. Li, H. Yang, L. Cai, and L. Gui, "A low-complexity equalization technique for OFDM system in time-variant multipath channels," in Proc. IEEE Veh. Technol. Conf., Mar. 2003, pp. 2466-2470.

[3] T. Wang, J. G. Proakis, and J. R. Zeidler, "Techniques for suppression of intercarrier interference in OFDM systems," in Proc. IEEE Wireless Commun. Netw. Conf., Mar. 2005, pp. 39-44.

[4] W. G. Jeon, K. H. Chang, and Y. S. Cho, "An equalization technique for orthogonal frequency division multiplexing systems in time-variant multipath channels," IEEE Trans. Commun., vol. 47, no. 1, pp. 27-32, Jan. 1999.

[5] Y. Choi, P. Voltz, and F. Cassara, "On channel estimation and detection for multicarrier signals in fast and selective Rayleigh fading channels," IEEE Trans. Commun., vol. 49, no. 8, pp. 1375-1387, Aug. 2001.

[6] X. Cai and G. Giannakis, "Bounding performance and suppressing intercarrier interference in wireless mobile OFDM," IEEE Trans. Commun., vol. 51, no. 12, pp. 2047-2056, Dec. 2003.

[7] K. Kim and H. Park, "A low complexity ICI cancellation method for high mobility OFDM systems," in Proc. IEEE Veh. Technol. Conf., May 2006, pp. $2528-2532$.

[8] A. F. Molisch, M. Toeltsch, and S. Vermani, "Iterative methods for cancellation of intercarrier interference in OFDM systems," IEEE Trans. Veh. Technol., vol. 56, no. 4, pp. 2158-2167, Jul. 2007.

[9] S. Chen and Y. Yang, "Low-complexity MMSE-SIC equalizer employing $L D L^{H}$ factorization for OFDM systems over time-varying channels," IEEE Trans. Vehicular Technol., vol. 59, no. 8, pp. 4128-1431, Oct. 2010.

[10] M.-L. Ku, W.-C. Chen, and C.-C. Huang, "EM-based iterative receivers OFDM and BICM/OFDM systems in doubly selective channels," IEEE Trans. Wireless Commun., vol. 10, no. 5, pp. 1405-1415, May 2011.

[11] S. Tomasin, A. Gorokhov, H. Yang, and L. P. Linnartz, "Iterative interference cancellation and channel estimation for mobile OFDM," IEEE Trans. Wireless Commun., vol. 4, no. 1, pp. 238-245, Jan. 2005.

[12] S.-J. Hwang and P. Schniter, "Efficient sequence detection of multi-carrier transmissions over doubly dispersive channels," EURASIP J. Appl. Signal Process., vol. 2006, pp. 93 638-1-93 638-17, 2006.

[13] X. Huang and H.-C. Wu, "Robust and efficient intercarrier interference mitigation for OFDM systems in time-varying fading channels," IEEE Trans. Veh. Technol., vol. 56, no. 5, pp. 2517-2528, Sep. 2007.

[14] Y. Mostofi and D. C. Cox, "ICI mitigation for pilot-aided OFDM mobile systems," IEEE Trans. Wireless Commun., vol. 4, no. 2, pp. 765-774, Mar. 2005.

[15] P. Schniter, "Low-complexity equalization of OFDM in doubly selective channels," IEEE Trans. Signal Process., vol. 52, no. 4, pp. 1002-1011, Apr. 2004.

[16] L. Rugini, P. Banelli, and G. Leus, "Low-complexity banded equalizers for OFDM systems in Doppler spread channels," EURASIP J. Appl. Signal Process., vol. 2006, pp. 67404-1-67404-1, Aug. 2006.

[17] S. Das and P. Schniter, "Max-SINR ISI/ICI-shaping multicarrier communication over the doubly dispersive channel," IEEE Trans. Signal Proccess., vol. 55, no. 12, pp. 5782-5795, Dec. 2007.

[18] F. Peng and W. Ryan, "A low-complexity soft demapper for OFDM fading channels with ICI," in Proc. IEEE Wireless Commun. and Networking Conf., 2006, pp. 1549-1554.

[19] P. Ochandiano, H. Wymeersch, M. Mendicute, L. Martinez, and I. Sobron, "Factor graph based detection approach for high-mobility OFDM systems with large FFT sizes," EURASIP J. Wireless Commun. Netw., pp. 16871499 , Oct. 2012.

[20] M.-X. Chang, "A novel algorithm of inter-subchannel interference selfcancellation in OFDM systems," IEEE Trans. Wireless Commun., vol. 6 , no. 8, pp. 2881-2893, Aug. 2007.

[21] C.-R. Sheu, M.-C. Tseng, C.-Y. Chen, and H.-P. Lin, "A low-complexity concatenated ICI cancellation scheme for high-mobility OFDM systems," in Proc. IEEE Wireless Commun. Netw. Conf., Mar. 2007, pp. 1389-1393.

[22] C.-R. Sheu, J.-W. Liu, and C.-C. Huang, "A low-complexity ICI cancellation scheme with multi-step windowing and modified SIC for highmobility OFDM systems," in Proc. IEEE Veh. Technol. Conf., May 2010, pp. $1-5$.

[23] M. Faulkner, L. R. Wilhelmsson, and J. Svensson, "Low-complex ICI cancellation for improving Doppler performance in OFDM systems," in Proc. IEEE Veh. Technol. Conf., Sep. 2006, pp. 1-5.

[24] K.-Y. Lin, H.-P. Lin, and M.-C. Tseng, "An equivalent channel time variation mitigation scheme for ICI reduction in high-mobility OFDM systems," IEEE Trans. Broadcast., vol. 58, no. 3, pp. 472-479, Sep. 2012.

[25] C.-Y. Ma, S.-W. Liu, and C.-C. Huang, "On optimum segment combining weight for ICI self-cancellation in OFDM systems under doubly selective fading channels," in Proc. IEEE Veh. Technol. Conf., May 2012, pp. 1-5.

[26] J.-C. Lin, "Least-squares channel estimation for mobile OFDM communication on time-varying frequency-selective fading channels," IEEE Trans. Veh. Technol., vol. 57, no. 6, pp. 3538-3550, Nov. 2008.

[27] O. Edfors, M. Sandell, J. J. van de Beek, S. K. Wilson, and P. O. Borjesson, "Analysis of DFT-based channel estimators for OFDM," Wireless Personal Commun., vol. 12, no. 1, pp. 55-70, Jan. 2000.

[28] S. Boyd and L. Vandenberghe, Convex Optimization. New York, NY, USA: Cambridge Univ. Press, 2004.

[29] S. M. Kay, Fundamentals of Statistical Signal Processing: Estimation Theory. Englewood Cliffs, NJ, USA: Prentice-Hall PTR, 1998.

[30] S. Chen and C. Zhu, "ICI and ISI analysis and mitigation for OFDM systems with insufficient cyclic prefix in time-varying channels," IEEE Trans. Consum. Electron., vol. 50, no. 1, pp. 78-83, Feb. 2004.

[31] C.-J. Park and G.-H. Im, "Efficient DMT/OFDM transmission with insufficient cyclic prefix," IEEE Commun. Lett., vol. 8, no. 9, pp. 576-578, Sep. 2004.

[32] C.-R. Sheu and C.-C. Huang, "A novel guard interval based ISI-free sampling region detection method for OFDM systems," in Proc. IEEE Veh. Technol. Conf., Sep. 2004, pp. 515-519.

[33] K. Ramasubramanian and K. Baum, "An OFDM timing recovery scheme with inherent delay-spread estimation," in Proc. IEEE GLOBECOM, Nov. 2001, vol. 5, pp. 3111-3115.

[34] J. Ma, P. V. Orlik, J. Zhang, and G. Y. Li, "Reduced-rate OFDM transmission for inter-subchannel interference self-cancellation over highmobility fading channels," IEEE Trans. Wireless Commun., vol. 11, no. 6, pp. 2013-2023, Jun. 2012.

[35] W. C. Jakes, Microwave Mobile Communications. New York, NY, USA: Wiley, 1974

[36] G. Golub and C. van Loan, Matrix Computations., 3rd ed. Baltimore, MD, USA: Johns Hopkins Univ. Press, 1996.

[37] F. Hlawatsch and G. Matz, Wireless Communications over Rapidly TimeVarying Channels. Amsterdam, The Netherlands: Elsevier, 2010.

[38] Digital Video Broadcasting (DVB); Framing Structure, Channel Coding and Modulation for Digital Terrestrial Television, ETSI EN 300 744 V1.6.1 (2009-1), 2009, European Telecommunications. Standards Institute.

[39] Guidelines for the Evaluation of Radio Transmission Technologies for IMT-2000, Recommendation ITU-R M.1225, 1997.

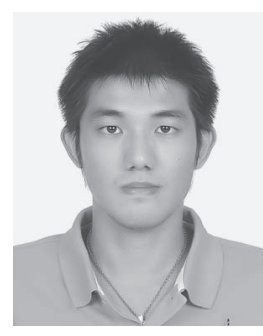

algorithms
Chun-Ying Ma (S'12) was born in Tainan, Taiwan, in 1985. He received the B.Eng. degree in communications from National Chiao Tung University, Hsinchu, Taiwan, in 2008. He is currently working toward the Ph.D. degree in wireless communications with the Department of Electrical and Computer Engineering, National Chiao Tung University.

His current research interests include highmobility orthogonal frequency-division multiplexing communication systems, green radio, wireless resource allocation, and phase noise suppression 


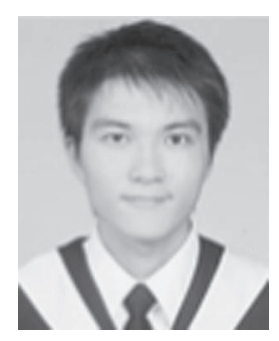

Sheng-Wen Liu was born in Taiwan. He received the B.S. and M.S. degrees in communications engineering from National Chiao Tung University, Hsinchu, Taiwan, in 2009 and 2011, respectively.

$\mathrm{He}$ is currently a System Application Engineer for touch panel integrated circuits with Novatek Microelectronics Corporation, Hsinchu.

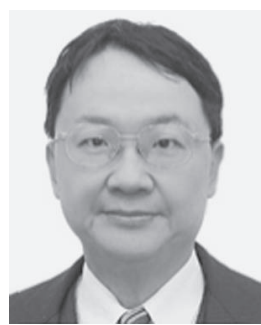

Chia-Chi Huang was born in Taiwan. He received the B.S. degree from National Taiwan University, Taipei, Taiwan, in 1977 and the M.S. and Ph.D. degrees from the University of California, Berkeley, CA, USA, in 1980 and 1984, respectively, all in electrical engineering.

From 1984 to 1988, he was a Radio-Frequency and Communication System Engineer with the Corporate Research and Development Center, General Electric Company, Schenectady, NY, USA, where he worked on mobile radio communication system design. From 1989 to 1992, he was a Research Staff Member with the IBM T.J. Watson Research Center, Yorktown Heights, NY, USA, working on indoor radio communication system design. Since 1992, he has been with National Chiao Tung University, Hsinchu, Taiwan, where he is currently a Professor with the Department of Electrical and Computer Engineering. 